\title{
REAL VS. COMPLEX RATIONAL CHEBYSHEV APPROXIMATION ON AN INTERVAL
}

BY

\author{
LLOYD N. TREFETHEN ${ }^{1}$ AND MARTIN H. GUTKNECHT
}

\begin{abstract}
If $f \in C[-1,1]$ is real-valued, let $E^{r}(f)$ and $E^{c}(f)$ be the errors in best approximation to $f$ in the supremum norm by rational functions of type $(m, n)$ with real and complex coefficients, respectively. It has recently been observed that $E^{c}(f)<E^{r}(f)$ can occur for any $n \geqslant 1$, but for no $n \geqslant 1$ is it known whether $\gamma_{m n}=\inf _{f} E^{c}(f) / E^{r}(f)$ is zero or strictly positive. Here we show that both are possible: $\gamma_{01}>0$, but $\gamma_{m n}=0$ for $n \geqslant m+3$. Related results are obtained for approximation on regions in the plane.
\end{abstract}

1. Introduction. Let $I$ be the unit interval $[-1,1], C^{r}$ the set of continuous real functions on $I$, and $\|\cdot\|$ the supremum norm $\|f\|=\sup _{x \in I}|f(x)|$. For nonnegative integers $m$ and $n$, let $R_{m n}$ and $R_{m n}^{r} \subseteq R_{m n}$ be the spaces of rational functions of type $(m, n)$ with coefficients in $\mathbf{C}$ and $\mathbf{R}$, respectively. For $f \in C^{r}$, let $E^{c}(f)$ and $E^{r}(f)$ denote the infima

$$
E^{c}(f)=\inf _{r \in R_{m n}}\|f-r\|, \quad E^{r}(f)=\inf _{r \in R_{m n}^{r}}\|f-r\| .
$$

It is known that both limits are attained, and a function that does so is called a best approximation $(B A)$ to $f$. In the real case the $\mathrm{BA}$ is unique [8], and in the complex case for $n \geqslant 1$ in general it is not $[7,10,11,14,15]$.

Obviously $E^{c} \leqslant E^{r}$ for any $f$, but since $f$ is real, it is not at first obvious whether a strict inequality can occur. However in 1971 Lungu [7], following a proposal of Gončar [16], published a class of examples showing that $E^{c}(f)<E^{r}(f)$ is indeed possible if $n \geqslant 1$. Independently, Saff and Varga [10,11] made the same discovery in 1977, and obtained more general sufficient conditions for $E^{c}(f)<E^{r}(f)$ and also a sufficient condition for $E^{c}(f)=E^{r}(f)$. The former was later sharpened by Ruttan [18] to the following statement: $E^{c}(f)<E^{r}(f)$ must hold if the best real approximation to $f$ attains its maximum error on no alternation set of length greater than $m+n+1$ points. For a survey of such results, see [14].

But is $E^{c}$ ever much less than $E^{r}$ ? If $\gamma_{m n}$ denotes the infimum

$$
\gamma_{m n}=\inf _{f \in C^{r} \backslash R_{m n}^{r}} E^{c}(f) / E^{r}(f),
$$

then one would like to know whether $\gamma_{m n}$ can be zero or is always positive, and if the latter, how small it is. In all of the examples devised to date, $E^{c}(f) / E^{r}(f)$ has fallen

Received by the editors September 3, 1982.

1980 Mathematics Subject Classification. Primary 30E10; Secondary 41A20, 41A25, 41A50.

Key words and phrases. Chebyshev approximation, rational approximation.

'Supported by a National Science Foundation Mathematical Sciences Postdoctoral Fellowship.

$$
\text { (C)1983 American Mathematical Society }
$$
$0002-9947 / 83 \$ 1.00+\$ .25$ per page 
in the range $\left(\frac{1}{2}, 1\right]$, suggesting that $\gamma_{m n}=\frac{1}{2}$ might be the minimum value. Saff and Varga posed in particular the question, is $\gamma_{n n}$ positive or zero [10,11]? Ellacott has suggested that $\gamma_{m n}=\frac{1}{2}$ may hold for $m \geqslant n$ [3]. (For more on his argument see §2.) Some partial results for $(m, n)=(1,1)$ have been obtained by Bennet, et al. $[1,2]$ and by Ruttan [9].

In this paper we resolve some of these questions, as follows. First, not only can $\gamma_{m n}<\frac{1}{2}$ occur, but $\gamma_{m n}=0$ for all $m \geqslant 0, n \geqslant m+3$ (Theorem 1). Second, $\gamma_{01}>0$ (Theorem 2). We conjecture that $\gamma_{m n}>0$ holds whenever $n<m+3$. Finally, at least some of our arguments extend to approximation on complex regions, and we show: $\gamma_{0 n}^{\Delta}=0$ for $n \geqslant 4$ in approximation on the unit disk $\Delta$ (Theorem 3). A similar result is obtained for approximation on a symmetric Jordan region.

2. $\gamma_{m n}=0$ for $n \geqslant m+3$.

THEOREM 1. $\gamma_{m n}=0$ for all $m \geqslant 0, n \geqslant m+3$.

Proof. The idea of the construction is indicated in Figure 1, where crosses represent poles and circles represent zeros.

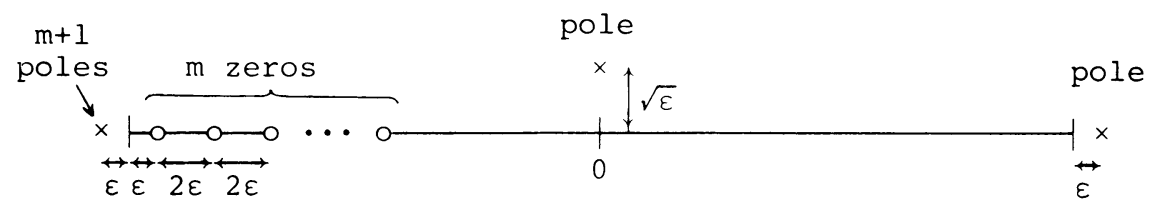

FIGURE 1

Given $m \geqslant 0$, let $\phi \in R_{m, n+3}$ be defined by

$$
\phi(x)=\frac{\varepsilon \prod_{j=1}^{m}[(-1+(2 j-1) \varepsilon)-x]}{[x+(1+\varepsilon)]^{m+1}[i \sqrt{\varepsilon}-x][(1+\varepsilon)-x]}
$$

and as the function in $C^{r}$ to be approximated take $f(x)=\operatorname{Re} \phi(x)$. We will show that $f$ has the following two properties:

(a) $\|f-\phi\|=\|\operatorname{Im} \phi\|=O(\sqrt{\varepsilon})$ as $\varepsilon \rightarrow 0$.

(b) There exists a constant $C>0$ such that for all sufficiently small $\varepsilon$,

$$
(-1)^{j} f(-1+2 j \varepsilon) \geqslant C, \quad 0 \leqslant j \leqslant m,
$$

and

$$
(-1)^{m+1} f(1) \geqslant C \text {. }
$$

Condition (b) states that the error function for the zero approximation to $f$ approximately equioscillates at $m+2$ points, and by the de la Vallee Poussin theorem for real rational approximation [8, Theorem 98], this implies $E^{r} \geqslant C$. (For the purposes of this theorem $r \equiv 0$ has rational type $(\mu, \nu)=(-\infty, 0)$, so the "defect" $d=\min \{m-\mu, n-\nu\}$ is $n$, which means one needs approximate equioscillation at $m+n+2-d=m+2$ points.) On the other hand if $n \geqslant m+3$, then $\phi \in R_{m n}$, so (a) implies $E^{c}=O(\sqrt{\varepsilon})$. Thus since $\varepsilon$ can be arbitrarily small, the theorem will be proved once (a) and (b) are established. 
Proof OF (a). Let us write $\phi$ as a product of three functions $\phi_{1}, \phi_{2}, \phi_{3}$ corresponding to the poles and zeros near $-1,0$, and 1 , respectively. Of these functions only $\phi_{2}$ has a nonzero imaginary part on $I$, and we bring this into the numerator. The factor $\phi_{1}$ gets the constant $\varepsilon$ from (3):

$$
\begin{aligned}
\phi(x) & =\phi_{1}(x) \phi_{2}(x) \phi_{3}(x) \\
& =\left(\frac{\varepsilon \prod_{j=1}^{m}[(-1+(2 j-1) \varepsilon)-x]}{[x+(1+\varepsilon)]^{m+1}}\right)\left(\frac{-i \sqrt{\varepsilon}-x}{x^{2}+\varepsilon}\right)\left(\frac{1}{(1+\varepsilon)-x}\right) .
\end{aligned}
$$

Since $(f-\phi)(x)=-i \operatorname{Im} \phi(x)$, we compute

$$
(f-\phi)(x)=-i \phi_{1}(x) \operatorname{Im} \phi_{2}(x) \phi_{3}(x)=\phi_{1}(x) \frac{i \sqrt{\varepsilon}}{x^{2}+\varepsilon} \phi_{3}(x) .
$$

It is not hard to see that on $\left[-1,-\frac{1}{2}\right]$ these factors have magnitude $O(1), O(\sqrt{\varepsilon})$, and $O(1)$, so their product is $O(\sqrt{\varepsilon})$. Similarly in $\left[-\frac{1}{2}, \frac{1}{2}\right]$ one has $O(\varepsilon) O(1 / \sqrt{\varepsilon}) O(1)=$ $O(\sqrt{\varepsilon})$, and in $[! \vdots, 1], O(\varepsilon) O(\sqrt{\varepsilon}) O(1 / \varepsilon)=O(\sqrt{\varepsilon})$. Together these estimates give $(f-\phi)(x)=O(\sqrt{\varepsilon})$ for all $x \in I$, as claimed.

Proof of (b). Again we use the factorization $\phi=\phi_{1} \phi_{2} \phi_{3}$ of (6). Let $\left\{x_{j}\right\}_{j=0}^{m}$ be the set of points $x_{j}=-1+2 j \varepsilon$ that appear in condition (4). At each $x_{j}, \phi_{1}$ evidently takes the form $\alpha_{j} \varepsilon^{m+1} / \beta_{j} \varepsilon^{m+1}$ for some constants $\alpha_{j}$ and $\beta_{j}$, and thus $\phi_{1}\left(x_{j}\right)$ is independent of $\varepsilon$. Moreover these quantities obviously alternate in sign, i.e.

$$
\phi_{1}\left(x_{0}\right)=\tau_{0}>0,-\phi_{1}\left(x_{1}\right)=\tau_{1}>0, \ldots,(-1)^{m} \phi_{1}\left(x_{m}\right)=\tau_{m}>0,
$$

with $\tau_{j}$ independent of $\varepsilon$. In addition since all of the points $x_{j}$ are contained in $[-1,-1+2 m \varepsilon]$. we have $\phi_{2}\left(x_{j}\right)=1+O(\sqrt{\varepsilon}), \phi_{3}\left(x_{j}\right)=\frac{1}{2}+O(\varepsilon)$ on $\left\{x_{j}\right\}$. Together these facts establish (4) for some $C=C_{1}>0$.

For condition (5) we compute

$$
\begin{aligned}
\phi(1) & =\phi_{1}(1) \phi_{2}(1) \phi_{3}(1) \\
& =\left(\frac{\varepsilon}{2}(-1)^{m}(1+O(\varepsilon))\right)(-1+O(\sqrt{\varepsilon})) \frac{1}{\varepsilon}=\frac{1}{2}(-1)^{m+1}+O(\sqrt{\varepsilon}),
\end{aligned}
$$

which implies that (5) holds for $C=C_{2}$ with any $C_{2}<\frac{1}{2}$. Taking $C=\min \left\{C_{1}, C_{2}\right\}$ now yields (b).

Remark on aN ARgument of Ellacott. As alluded to in the Introduction, Ellacott has observed that one can conclude from the $C F$ method $[13,4]$ that if $p$ is a polynomial of degree $m+1$, then

$$
E^{c}(p) / E^{r}(p) \geqslant \frac{1}{2}
$$

for $n \leqslant m$ [3]. This is one of his arguments for suggesting that $\gamma_{m n}=\frac{1}{2}$ or at least $\gamma_{m n}>0$ may hold for $n \leqslant m$. However we claim that (7) is valid in fact for all $n \leqslant 2 m+1$, which by Theorem 1 means that it holds even in many cases with $\gamma_{m n}=0$. Therefore although Ellacott's conjecture is plausible, it appears that (7) does not provide very strong support for it. 
To demonstrate that (7) holds for $n \leqslant 2 m+1$, let $p$ be transplanted to the unit circle by defining a function $\hat{p}$ for $z \in \mathbf{C}$ as follows:

$$
x=\frac{1}{2}\left(z+z^{-1}\right), \quad \hat{p}(z)=p(x)=p\left(\frac{1}{2} z+\frac{1}{2} z^{-1}\right)=\sum_{k=-m-1}^{m+1} \alpha_{k} z^{k} .
$$

For $n \leqslant 2 m+1$, the BA to $p$ in $R_{m n}^{r}$ on $I$ was obtained explicitly by Talbot $[12,5]$, and its deviation from $p$ is

$$
E^{r}(p)=2 \sigma_{n}
$$

where $\sigma_{n}$ is the smallest singular value of the $(n+1) \times(n+1)$ Hankel matrix $\left(\alpha_{m-n+1+i+j}\right)_{i, j=0}^{n}$. On the other hand if $r \in R_{m n}$ is any complex approximation to $p$ on $I$, consider the transplanted function $\hat{r}$ defined by $\hat{r}(z)=r(x)$. It is readily verified that $\hat{r}$ has $\nu \leqslant n$ poles in $1<|z|<\infty$ and is of order $O\left(z^{m-\nu}\right)$ at $\infty$. Therefore $\hat{r}$ lies in the space $\tilde{R}_{m n}$ defined in $[13,4]$, and by the theory given there this implies

$$
\sigma_{n} \leqslant \sup _{|z|=1}|(\hat{p}-\hat{r})(z)|=\sup _{|x|=1}|(p-r)(x)| .
$$

Thus

$$
E^{c}(p) \geqslant \sigma_{n},
$$

which together with (8), establishes (7).

By applying [4, Lemma 5.1 in Part II] (7) can be seen to hold even for some rational functions $f$, namely for those of exact type $(M, N)$ where either $M \leqslant m+1$, $N=n+1, n \leqslant m$ or $M=m+1, N \leqslant n+1, n \leqslant 2 m+1-N$; details will be given in [5].

3. $\gamma_{01}>0$.

THEOREM 2. $\gamma_{01}>0$.

Proof. Let $f \in C^{r}$ be arbitrary, and let $c^{*}$ be a BA to $f$ in $R_{m n}$. Then for any $r \in R_{m n}^{r}$ one has $\left\|\operatorname{Im} c^{*}\right\| \leqslant\left\|f-c^{*}\right\|=E^{c}(f)$ and $E^{r}(f) \leqslant E^{c}(f)+\left\|c^{*}-r\right\|$, and therefore

$$
E^{r}(f) \leqslant E^{c}(f)+\left\|\operatorname{Im} c^{*}\right\| \frac{\left\|c^{*}-r\right\|}{\left\|\operatorname{Im} c^{*}\right\|} \leqslant E^{c}(f)\left(1+\frac{\left\|c^{*}-r\right\|}{\left\|\operatorname{Im} c^{*}\right\|}\right) .
$$

Now suppose that for any $c \in R_{m n} \backslash R_{m n}^{r}$ with no poles on $I$, one can find $r^{(c)} \in R_{m n}^{r}$ such that

$$
\left\|c-r^{(c)}\right\| /\|\operatorname{Im} c\| \leqslant M
$$

for some fixed $M$. Then $r^{\left(c^{*}\right)}$ can be inserted in (10), independent of $f$, and one obtains $\gamma_{m n} \geqslant 1 /(1+M)$. Our proof of $\gamma_{01}>0$ consists of exhibiting a mapping $c \mapsto r^{(c)}$ for the case $(m, n)=(0,1)$ that satisfies $(11)$.

Thus let $c(z)=a /\left(1-z / z_{0}\right)$ be given, where $z_{0}$ lies in the region $C^{0}=$ $\mathrm{C} \cup\{\infty\} \backslash I$. Let $\theta \in(0, \pi / 2)$ and $\rho \in(1, \infty)$ be arbitrary fixed constants (say, 
$\theta=\pi / 4, \rho=2$ ). Our choice of $r^{(c)}$ depends on which of four domains $A^{+}, A^{-}, B, C$ the pole lies in:

$$
\begin{aligned}
A^{ \pm} & =\{z \in C:|\arg (-1 \pm z)|<\theta\}, \\
B & =\left\{z \in C-A^{+}-A^{-}:|z| \leqslant \rho\right\}, \\
C & =C^{0}-A^{+}-A^{-}-B .
\end{aligned}
$$

The configuration is indicated in Figure 2.

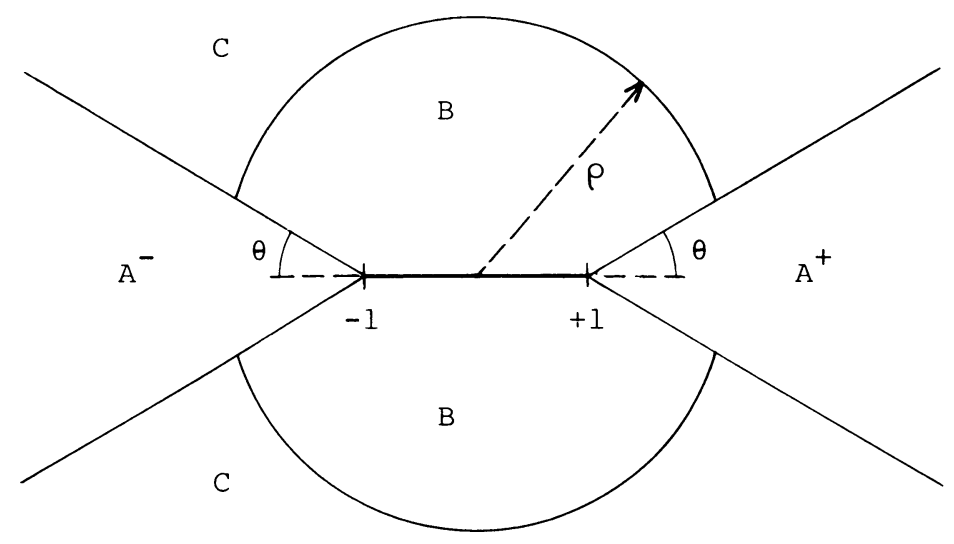

FIGURE 2

We define $r^{(c)}$ as follows:

$$
\begin{array}{ll}
\text { For } z_{0} \in A^{ \pm}: & r^{(c)}(z)=\frac{1-1 /\left|z_{0}\right|}{1 \mp z /\left|z_{0}\right|} \operatorname{Re} c( \pm 1) . \\
\text { For } z_{0} \in B: & r^{(c)} \equiv 0 . \\
\text { For } z_{0} \in C: & r^{(c)} \equiv \operatorname{Re} a .
\end{array}
$$

The proof can now be completed by showing that there exist constants $M_{A}, M_{B}, M_{C}$ such that (11) holds for $z_{0}$ restricted to each domain $A^{+} \cup A^{-}, B, C$. The global constant $M$ can then be taken as $M=\max \left\{M_{A}, M_{B}, M_{C}\right\}$. The algebra involved is unfortunately quite tedious, so we will omit these verifications. However, details of a similar argument for the case of approximation on certain Jordan regions in $\mathbf{C}$ are given in [17].

\section{4. $\gamma_{0 n}^{\Delta}=0$ for $n \geqslant 4$.}

Let $\Delta$ be the closed unit disk $\{z \in \mathbf{C}:|z| \leqslant 1\}$, and let $f$ be continuous in $\Delta$ and analytic in the interior and satisfy $f(\bar{z})=\overline{f(z)}$. Let $\|f\|_{\Delta}$ denote $\sup _{z \in \Delta}|f(z)|$, and define $E^{c}(f ; \Delta), E^{r}(f ; \Delta)$, and $\gamma_{m n}^{\Delta}$ as in (1) and (2). Until recently it was not even known whether $\gamma_{m n}^{\Delta}<1$ is possible, but in a separate paper we show that this inequality holds at least for all pairs $(m, n)$ with $m=0, n \geqslant 1$ or $m \geqslant 0, n=1$ [6].

By a variation of the argument of $\S 2$, we will now prove

THEOREM 3. $\gamma_{0 n}^{\Delta}=0$ for $n \geqslant 4$. 
Proof. Let $\zeta=e^{i \theta}$ for some fixed $\theta \in(0, \pi)$, and for any $\varepsilon>0$, define

$$
\phi(z)=\frac{\varepsilon(1-\zeta)^{2}}{[z+(1+\varepsilon)][(1+\varepsilon)-z]\left[z-\left(1+\varepsilon^{1 / 3}\right) \zeta\right]^{2}}
$$

and

$$
f(z)=\frac{1}{2}(\phi(z)+\overline{\phi(\bar{z})}) .
$$

In analogy to the proof of Theorem $1, \gamma_{0 n}^{\Delta}=0$ for $n \geqslant 4$ will follow from the properties

(a) $\|f-\phi\|_{\Delta}=O\left(\varepsilon^{1 / 3}\right)$;

(b) there exists a constant $C>0$ such that for all sufficiently small $\varepsilon, f(-1) \leqslant-C$, $f(1) \geqslant C$.

Both (a) and (b) can be readily derived by observing that the term

$$
(1-\zeta)^{2} /\left[z-\left(1+\varepsilon^{1 / 3}\right) \zeta\right]^{2}
$$

behaves like $1+O\left(\varepsilon^{1 / 3}\right)$ near $z=1$ and like $-|(1-\zeta) /(1+\zeta)|^{2}+O\left(\varepsilon^{1 / 3}\right)$ near $z=-1$. We omit the details.

This argument can be extended to show $\gamma_{0 n}^{\Omega}=0$ for $n \geqslant 4$ for approximation on any Jordan region $\Omega$ with $\Omega=\bar{\Omega}$, provided $\partial \Omega$ is differentiable at its two points of intersection with $\mathbf{R}$, say $z_{1}$ and $z_{2}$, hence forms a right angle to $\mathbf{R}$ at these points. Again one introduces a complex double pole, slightly above the point $z_{1}$ (analogous to taking $\xi=e^{i \theta}$ with $\theta$ small above), and this generates an approximate sign change between $\phi\left(z_{1}\right)$ and $\phi\left(z_{2}\right)$.

One can also prove $\gamma_{01}^{\Omega}>0$ for the same class of regions $\Omega$. See [17].

Note added in proof. After studying the present paper, E. Saff has pointed out to us that the existence of arbitrarily small numbers $\gamma_{m n}$ is implied by a result of Walsh in 1934 [19, Theorem IV], although this consequence was never recognized. Walsh showed that for any $m \geqslant 0$, the family $\cup_{n=0}^{\infty} R_{m n}$ is dense in $C[I]$ (or indeed in the space of continuous functions on any Jordon arc in $\mathbf{C})$, so that $\lim _{h \rightarrow \infty} E_{m n}(f)=0$ for $f \in C[I]$. On the other hand, as we have seen, if $f$ has $m+1$ zeros, then it cannot be approximated arbitrarily closely in $\cup_{n=0}^{\infty} R_{m n}^{r}$, i.e. $\lim _{n \rightarrow \infty} E_{m n}^{r}(f)>0$. It follows that for any $m \geqslant 0, \lim _{n \rightarrow \infty} \gamma_{m n}=0$.

\section{REFERENCES}

1. C. Bennet, K. Rudnick and J. D. Vaaler, On a problem of Saff and Varga concerning best rational approximation, Padé and Rational Approximation (E. B. Saff and R. S. Varga, eds.), Academic Press, New York, 1977, pp. 483-488.

2. Best uniform approximation by linear fractional transformations, J. Approximation Theory 25 (1979), 204-224.

3. S. W. Ellacott, $A$ note on a problem of Saff and Varga concerning the degree of complex rational approximation to real valued functions, Bull. Amer. Math. Soc. (N. S.) 6 (1982), 218-220.

4. M. H. Gutknecht, On complex rational approximation, Computational Aspects of Complex Analysis (H. Werner, et al., eds.), Reidel, Dordrecht, 1983.

5. Algebraically solvable Cheby'shev approximation problems, Approximation Theory IV (L. Schumaker, ed.), Academic Press, New York (in press).

6. M. H. Gutknecht and L. N. Trefethen, Nonuniqueness of rational Chebyshev approximations on the unit disk, J. Approximation Theory (in press). 
7. K. N. Lungu, Best approximation by rational functions, Mat. Zametki 10 (1971), 11-15. (Russian)

8. G. Meinardus, Approximation of functions: Theory and numerical methods, Springer-Verlag, Berlin and New York, 1967.

9. A. Ruttan, On the cardinality of a set of best complex rational approximations to a real function, Pade and Rational Approximation (E. B. Saff and R. S. Varga, eds.), Academic Press, New York, 1977, pp. 303-319.

10. E. B. Saff and R. S. Varga, Nonuniqueness of hest approximating complex rational functions, Bull. Amer. Math. Soc. 83 (1977), 375-377.

11. Nonuniqueness of best complex rational approximations to real functions on real intervals, J. Approximation Theory 23 (1978), 78-85.

12. A. Talbot, On a class of Tchebysheffian approximation problems solvable algebraically, Proc. Cambridge Philos. Soc. 58 (1962), 244-267.

13. L. N. Trefethen, Rational Chebyshev approximation on the unit disk, Numer. Math. 37 (1981), $297-320$.

14. R. S. Varga, Topics in polynomial and rational interpolation and approximation, Les Presses de l'Université de Montréal, Montréal, 1982.

15. J. L. Walsh, Interpolation and approximation by rational functions in the complex domain, Amer. Math. Soc. Colloq. Publ., vol. 20, Amer. Math. Soc., Providence, R. I., 1935 (5th ed., 1969).

16. A. A. Gončar, The rate of approximation by rational fractions and the properties of functions, Proc. Internat. Congress of Mathematicians (Moscow, 1966), Izdat. "Mir”, Moscow, 1968. (Russian)

17. M. H. Gutknecht and L. N. Trefethen, Real vs. complex rational Chebyshev approximation on complex domains, Numerische Methoden der Approximationstheorie, vol. 7 (L. Collatz and H. Werner, eds.), Birkhäuser (in press).

18. A. Ruttan, The length of the alternation set as a factor in determining when a best real rational approximation is also a best complex rational approximation, J. Approx. Theory 31 (1981), 230-243.

19. J. L. Walsh, On approximation to an analytic function by rational functions of best approximation, Math. Z. 38 (1934), 163-176.

Courant Institute of Mathematical Sciences, New York University, New York, New York 10012

Seminar fUr Angewandte Mathematik, ETH, ZUrich, Switzerland 